\title{
Resonant PZT MEMS Scanner for High-Resolution Displays
}

\author{
Utku Baran, Student Member, IEEE, Dean Brown, Sven Holmstrom, Davide Balma, \\ Wyatt O. Davis, Member, IEEE, Paul Muralt, Senior Member, IEEE, and Hakan Urey, Senior Member, IEEE
}

\begin{abstract}
A resonant piezoelectric scanner is developed for high-resolution laser-scanning displays. A novel actuation scheme combines the principle of mechanical amplification with lead zirconate titanate (PZT) thin-film actuation. Sinusoidal actuation with $24 \mathrm{~V}$ at the mechanical resonance frequency of $40 \mathrm{kHz}$ provides an optical scan angle of $38.5^{\circ}$ for the 1.4-mm-wide mirror. This scanner is a significant step toward achieving full-highdefinition resolution $(1920 \times 1080$ pixels $)$ in mobile laser projectors without the use of vacuum packaging. The reported piezoscanner requires no bulky components and consumes $<$ 30-mW power at maximum deflection, thus providing significant power and size advantages, compared with reported electromagnetic and electrostatic scanners. Interferometry measurements show that the dynamic deformation is at acceptable levels for a large fraction of the mirror and can be improved further for diffraction-limited performance at full resolution. A design variation with a segmented electrode pair illustrated that reliable angle sensing can be achieved with PZT for closed-loop control of the scanner.

[2012-0116]
\end{abstract}

Index Terms-High-frequency laser beam scanning, lead zirconate titanate (PZT) thin-film-actuated, microelectromechanical systems (MEMS), MEMS mirror, resonant scanner.

\section{INTRODUCTION}

$\mathbf{M}$ ICROELECTROMECHANICAL systems (MEMS) scanners are widely used in displays [1]-[3], tunable lasers [4], optical switches [5], variable optical attenuators [6], spectroscopy [7], biomedical imaging [8], and laser printers [9]. Commercial interest for mobile picoprojectors has increased rapidly in recent years, driving the interest in high-performance scanners [3]. Such scanners for laser display applications typically require actuators that can provide a large scan angle with a large mirror at high frequency and a low driving voltage. The requirements for horizontal MEMS scanners for use in laser-scanning-based high-resolution display were reported in [10]. Single-crystal silicon is usually utilized at resonant mode operation to satisfy the requirements for frequency and scan angle.

Manuscript received May 3, 2012; revised July 7, 2012; accepted July 10, 2012. Date of publication August 10, 2012; date of current version November 27, 2012. This work was supported in part by Microvision Inc., USA, and in part by the FP7 project PiezoVolume. Subject Editor J. A. Yeh.

U. Baran, S. Holmstrom, and H. Urey are with the Optical Microsystems Laboratory, Department of Electrical and Electronics Engineering, Koç University, Istanbul 34450, Turkey (e-mail: hurey@ku.edu.tr).

D. Brown and W. O. Davis are with Microvision, Inc., Redmond, WA 98052 USA (e-mail: dean_brown@microvision.com; wyatt_davis@ microvision.com).

D. Balma and P. Muralt is with the Department of Material Science, École Polytechnique Fédérale de Lausanne, CH-1015 Lausanne, Switzerland.

Color versions of one or more of the figures in this paper are available online at http://ieeexplore.ieee.org.

Digital Object Identifier 10.1109/JMEMS.2012.2209405
The majority of best-performing scanners in the literature are either electromagnetically (EM) or electrostatically (ES) actuated. EM scanners require the placement of bulky off-chip magnets close to the scanning device, leading to increased package size. On the other hand, ES comb-drive scanners are fairly simple to fabricate and can be compact, but they require high drive voltages. In addition, several scanning MEMS mirrors actuated by applying voltage to stack lead zirconate titanate (PZT) layers have been reported [9]. In a few cases, this is done with an asymmetric design excited by an external PZT stack attached to the chip [11], [12]. However, it is difficult to fabricate such devices monolithically via the silicon wafer process, and stack PZT actuators consume space, as is the case with EM actuators. Hence, when key features for scanning MEMS mirrors, such as achieving large deflection with low voltage in a small package, are considered, exploring new actuation mechanisms remains an important and challenging task.

Piezoelectric actuation with thin-film PZT is a promising alternative due to its potential to offer equal performance at much lower voltage levels than ES scanners and its much smaller package size, compared with EM scanners. Significant research effort has been spent to develop piezoelectric MEMS for various applications [13] such as ultrasonic micromotors for watches [14], ultrasonic transducers [15], atomic force microscopy [16], and microscanners [17]-[27]. Moreover, piezoelectric actuators can measure deflection from the piezoelectric voltage of the separated electrode [28] or the piezoelectric current charge [29]. Thus, they can be used as angle sensors for closed-loop control of microscanners without any additional process or material. Although thin-film PZT actuation is a promising candidate to meet the requirements of both high power density and size for mobile laser projectors, mechanical design challenges still remain to be solved. For high-frequency resonant PZT scanners, damping losses at high frequencies and limited deflection of PZT beams with large stiffness are the two main obstacles to overcome.

In this paper, we introduce a novel actuation scheme that combines PZT thin-film actuation with the principle of mechanical amplification, previously used for EM-actuated and ES-actuated scanners [2], [30], to achieve high-performance PZT-actuated resonant MEMS scanners. Fig. 1 compares reported PZT thin-film-actuated scanner performances according to a combined figure of merit $\theta_{\mathrm{OPT}} \cdot D \cdot f$, which indicates the possible rate of pixels per seconds. $\theta_{\mathrm{OPT}}$ is the optical scan angle, $D$ is the mirror width along the scan direction, and $f$ is the scan frequency. The device design and fabrication processes are developed in Section II. The theoretical motivation and 


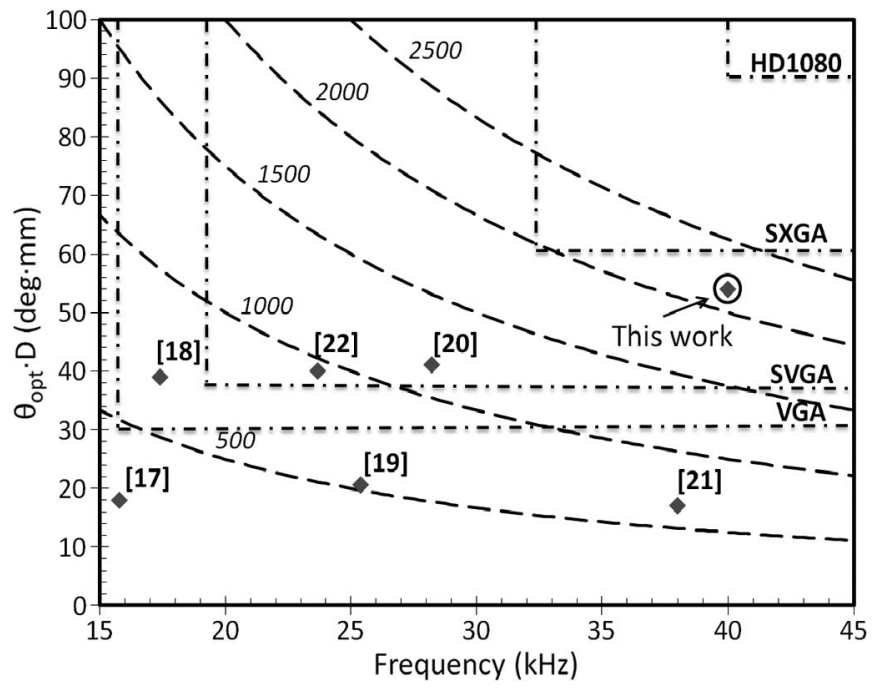

Fig. 1. Performance comparison of piezoelectric thin-film-actuated microscanners based on the literature. The dashed arcs indicate the selected levels of $\theta_{\mathrm{OPT}} \times D \times f$-products in deg $\times \mathrm{mm} \times \mathrm{kHz}$ for better comparison of scanners between different working frequencies. The dash-dotted squares show the requirements for different display formats, assuming $60-\mathrm{Hz}$ refresh rate and bidirectional scanning.

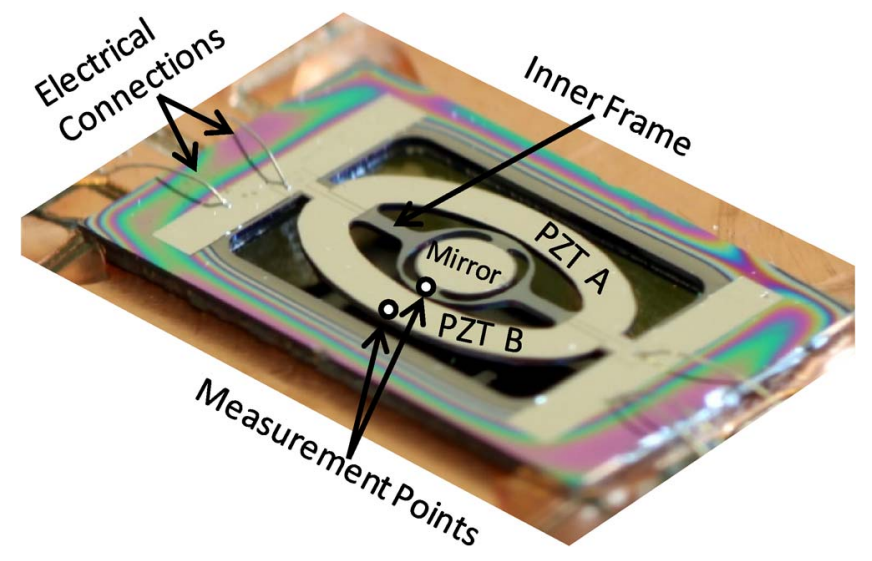

Fig. 2. Scanner wirebonded to a custom-made printed circuit board. The major structural components of the device are pointed out, as well as the two points used for amplitude measurements. PZT A and B are the complementary drive electrodes that together make up the outer frame. The inner frame is designed to mechanically isolate the mirror from the rotational flexure to minimize mirror deformation.

a finite-element model are described in Section III, and in Section IV, the device is characterized, and the results are reported. Finally, Section V provides concluding remarks.

\section{Device Properties}

\section{A. Device Design}

The presented scanner consists of two cascaded frames. The outer frame is the actuator and is referred to in Fig. 2 as PZT A-B. The inner frame holds the scanning mirror and is carefully designed to limit the dynamic deformation of the mirror. The outer frame is anchored to the substrate, and the inner frame is attached to the outer frame via torsional flexures. PZT thin-film actuators are placed on each side of the outer frame, as shown in Fig. 2. Any cascaded two-frame mechanical structure will have two separate rotation modes: the two frames rotate in phase, or they rotate out of phase. The present device is designed to rotate the large mirror at the out-of-phase resonance with large angular amplification.

There are two main advantages of a design using two cascaded frames operated out of phase, compared with a mechanism without an outer frame (i.e., a direct drive, where the PZT actuators are attached directly to the mirror). First, larger scan angles can be achieved by keeping the scan mirror and inner flexures small and free from additional stress-inducing layers. Second, limited deflection of the PZT film can be amplified to achieve large mirror rotation at resonance by placing the actuators on the outer frame, as shown in Fig. 2. The problem with the previously reported PZT actuators with long lever arms is achieving good mode separation without crosstalk and poor shock and vibration survivability due to long flexures. There is one reported PZT thin-film scanner by Tani et al. using a similar design with an outer frame to actuate an interior mirror; unfortunately, this paper lacks details of the actuation principle [26].

The $125-\mu \mathrm{m}$-thick device has a $3.75 \mathrm{~mm} \times 6.15 \mathrm{~mm}$ die size. The entire structure is anchored to the substrate with a main suspension system consisting of two $220-\mu \mathrm{m}$-long and $520-\mu \mathrm{m}$-wide flexures. The inner frame, in turn, is attached to the outer frame by a pair of $480-\mu \mathrm{m}$-long and $270-\mu \mathrm{m}$-wide flexures. To lower the peak stress, the corners of the connection points of the flexures are rounded. The scanner employs a $1 \mathrm{~mm} \times 1.4 \mathrm{~mm}$ oval mirror in the inner frame. Two main strategies are employed in order to diminish dynamic deformation of the mirror. A $175-\mu \mathrm{m}$-thick cross-shaped backside reinforcement rim is left from the handle layer silicon underneath the mirror to increase mirror stiffness. Second, the mirror is mechanically isolated from the rotational flexure by the isolating inner frame, as shown in Fig. 2.

\section{B. Fabrication}

The microfabrication is performed with a six-mask process using silicon-on-insulator (SOI) wafers having a $125-\mu \mathrm{m}$-thick device layer, as shown in Fig. 3. The first fabrication step is to thermally grow a $2-\mu$ m-thick $\mathrm{SiO}_{2}$ layer on both sides of the wafer. The bottom electrode was deposited on the oxidized wafers with a Balzers BAS 450 multitarget sputtering system at $300{ }^{\circ} \mathrm{C}$. It consists of a Pt/ $\mathrm{TiO}_{2} / \mathrm{i}$ stack with thicknesses of 100 , 50 , and $3 \mathrm{~nm}$, respectively. Ti was used as an adhesion layer, with $\mathrm{TiO}_{2}$ as a barrier layer to prevent the diffusion of titanium to the top surface of the platinum or also to prevent the reaction of lead with $\mathrm{SiO}_{2}$. The textured $\mathrm{Pt}(111)$ film serves as electrode and as growth seed layer for obtaining a highly $\{100\}$ c-textured $\mathrm{PbTiO}_{3}$ (PTO) film (20 nm thick) used as template for the growth of the subsequent 2- $\mu$ m-thick PZT $\{100\}$ film [31]. PTO and PZT were deposited by a sol-gel technique based on an improved 2-methoxyethanol route, as described in [31]. The PTO film was the result of one spin, one pyrolysis step, and one rapid thermal annealing step $\left(60 \mathrm{~s}\right.$ at $\left.650{ }^{\circ} \mathrm{C}\right)$. The $2-\mu \mathrm{m}$-thick PZT film was deposited with eight annealing steps $\left(650{ }^{\circ} \mathrm{C}\right)$, each consisting of four solution spinning $(4000 \mathrm{rev} / \mathrm{min}$ for $40 \mathrm{~s})$ and pyrolysis steps $\left(350{ }^{\circ} \mathrm{C}\right.$ for $\left.60 \mathrm{~s}\right)$. The four solutions had excess lead concentrations of $10 \%$ for the first three layers and $30 \%$ for the last layer to compensate for lead evaporation 
1)
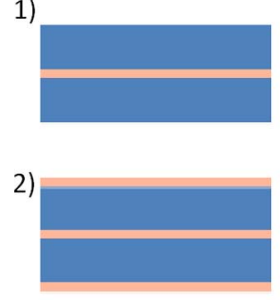

5)
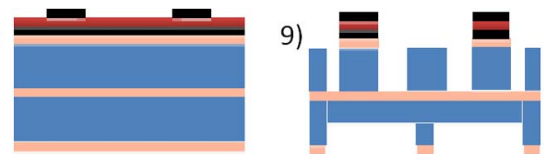

6)

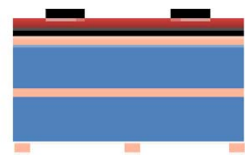

10)

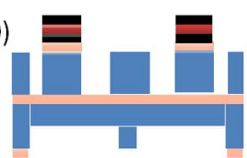

3)

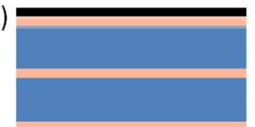

7)
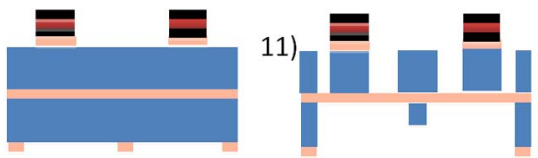

4

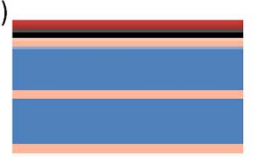

8)

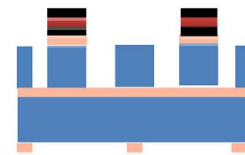

12)

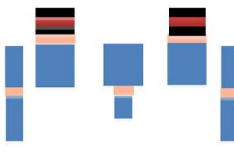

$\mathrm{Si}$

Ti/Pt PZT
Fig. 3. Detailed fabrication process of the device. (1-5) Starting with SOI wafer, deposition of $\mathrm{SiO}_{2}$, bottom electrode, PZT, and patterning of top electrode by liftoff. (6-8) Patterning of PZT with wet etch, and bottom electrode and $\mathrm{SiO}_{2}$ layers with RIE. (9-10) The front side silicon is shaped by DRIE, followed by the timed backside DRIE. (11-12) Final backside DRIE of Si and $\mathrm{SiO}_{2}$ to release the device.

during annealing. The temperature ramp during annealing was optimized in order to achieve the desired perosvkite phase with $\{100\}$ orientation. The average Ti-to- $\mathrm{Zr}$ ratio was chosen to be that of the morphotropic phase boundary, i.e., $\mathrm{Pb}\left(\mathrm{Zr}_{53} \mathrm{Ti}_{47}\right) \mathrm{O}_{3}$, in order to maximize the piezoelectric properties. The solutions could still be improved concerning the $\mathrm{Ti} / \mathrm{Zr}$ concentration homogeneity as described in [32].

As a last deposition step, a 100-nm-thick Pt top electrode layer was evaporated and patterned using a liftoff process on the PZT layer (Mask 1).

Next, the backside oxide is patterned with photolithography and dry etch (Mask 2). This patterning is later used to create backside mirror reinforcement. In order to protect the reinforcement areas beneath the mirror during backside silicon etch, the backside $\mathrm{SiO}_{2}$ layer is patterned, so that the $2-\mu$ m-thick $\mathrm{SiO}_{2}$ protective layer is left on the reinforcement areas. The desired geometry of the PZT was defined by patterning with $8-\mu \mathrm{m}$ thick AZ 9260 photoresist and wet etching (Mask 3). The 2- $\mu \mathrm{m}$ PZT layer is wet etched in a chemical solution consisting of $30 \%$ of DI water, $70 \%$ of chloride acid (diluted at $30 \%$ ), and a few droplets of hydrofluoric acid (diluted at $40 \%$ ). The etch rate was about $1 \mu \mathrm{m} / \mathrm{min}$. By contrast, after patterning the PZT film, the bottom electrode is etched in chlorine chemistry using an STS Multiplex ICP dry etcher after being defined through photolithography (Mask 4).

The front-side device structures are patterned and etched with deep reactive ion etch (DRIE) using the Bosch process (Mask 5). The backside is patterned with $10 \mu \mathrm{m}$ of AZ 9260 resist (Mask 6) and is then time etched with DRIE. When $175 \mu \mathrm{m}$ of $\mathrm{Si}$ has been etched from the backside using DRIE, the remaining $\mathrm{SiO}_{2}$ under the mirror is removed with dry etch. After completing the backside DRIE of Si, the devices are released by the final oxide etch. This six-mask fabrication
TABLE I

Material Parameters USEd For MOdEling

\begin{tabular}{|l|c|c|}
\hline Parameter & PZT & Si \\
\hline Density $\rho\left(\mathrm{kg} / \mathrm{m}^{3}\right)$ & 7500 & 2330 \\
\hline$C_{71}\left(10^{9} \mathrm{~N} / \mathrm{m}^{2}\right)$ & 127 & 166.1 \\
\hline$C_{12}\left(10^{9} \mathrm{~N} / \mathrm{m}^{2}\right)$ & 80.2 & 64.2 \\
\hline$C_{13}\left(10^{9} \mathrm{~N} / \mathrm{m}^{2}\right)$ & 84.6 & \\
\hline$C_{33}\left(10^{9} \mathrm{~N} / \mathrm{m}^{2}\right)$ & 117 & \\
\hline$C_{44}\left(10^{9} \mathrm{~N} / \mathrm{m}^{2}\right)$ & 23 & 79.4 \\
\hline$C_{55}\left(10^{9} \mathrm{~N} / \mathrm{m}^{2}\right)$ & 23.4 & \\
\hline$v$ & 0.38 & 0.28 \\
\hline
\end{tabular}

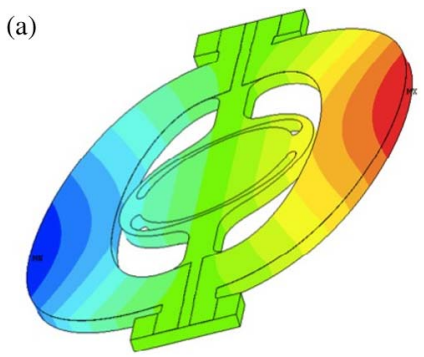

(b)

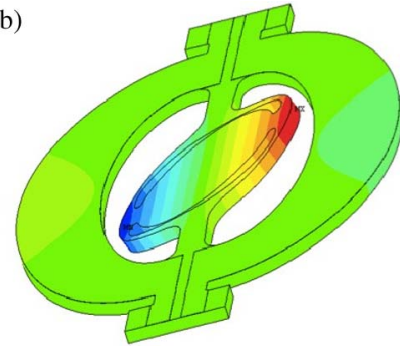

$\begin{array}{lllllllllllllllll}-954 & -700 & -445 & -190 & 63 & 318 & \frac{572}{572} & 954 & 2257 & -1655 & -1053 & -451 & 150 & 752 & 1354 & 2257\end{array}$

Fig. 4. Shapes of the mechanical operation eigenmodes of the microscanner. The scales use an arbitrary unit. (a) In-phase torsion mode, where the outer frame and inner frame motions are in phase at $15391-\mathrm{Hz}$ resonance frequency. (b) Out-of-phase torsion mode, where the outer frame and inner frame motions are out of phase with a rotation angle ratio of 17 at $39579-\mathrm{Hz}$ resonance frequency.

scheme leaves oxide on the mirror, leading to somewhat increased stress. The oxide could easily be removed by adding one additional mask to the process. Details on the impact of this choice can be found in Section IV.

\section{FEA}

\section{A. FEA Results}

Finite-element analysis (FEA) of the PZT scanner is performed using ANSYS software. For the sake of simplicity, only $\mathrm{Si}$ and PZT layers are modeled. Material properties are used as in Table I.

Modal analysis is done to extract the eigenmodes. As shown in Fig. 4, at the second mode, which is found to be at $15391 \mathrm{~Hz}$, the outer frame and inner frame motions are in phase, and at the fourth mode, which is found to be at $39579 \mathrm{~Hz}$, the respective motions of the inner and outer frames are out of phase. At the desired fourth mode, the mechanical coupling coefficient, which determines the rotation angle ratio of the outer and inner frames, is arranged to be 17 .

The presented device is designed to achieve $\pm 10^{\circ}$ mechanical scan angle at its desired resonance frequency. Fig. 5 shows that the maximum stress is $863 \mathrm{MPa}$ at $10^{\circ}$ and at resonance near $40 \mathrm{kHz}$. The stress is significantly below the silicon stress limit and observed at the connection of the inner flexure to the outer frame. Using ANSYS, the shape of the flexures was engineered, and connection areas with the outer frame were rounded to diminish the peak mechanical stress.

The dynamic deformation of the mirror is one of the essential properties of optical MEMS scanners that determine the quality of the reflected beam. Ideally, it should be less than $10 \%$ of 


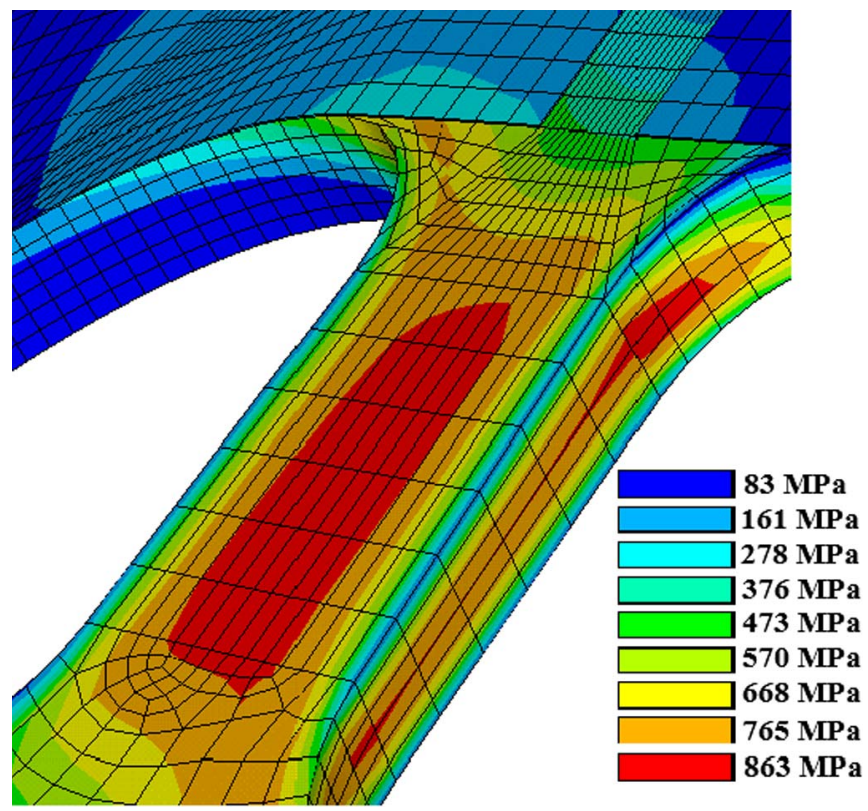

Fig. 5. Stress distribution of the torsional scanner. At the desired mode of the scanner, stress is accumulated at the inner flexure, and the maximum principal stress is found to be $863 \mathrm{MPa}$ at $10^{\circ}$ mechanical scan angle.

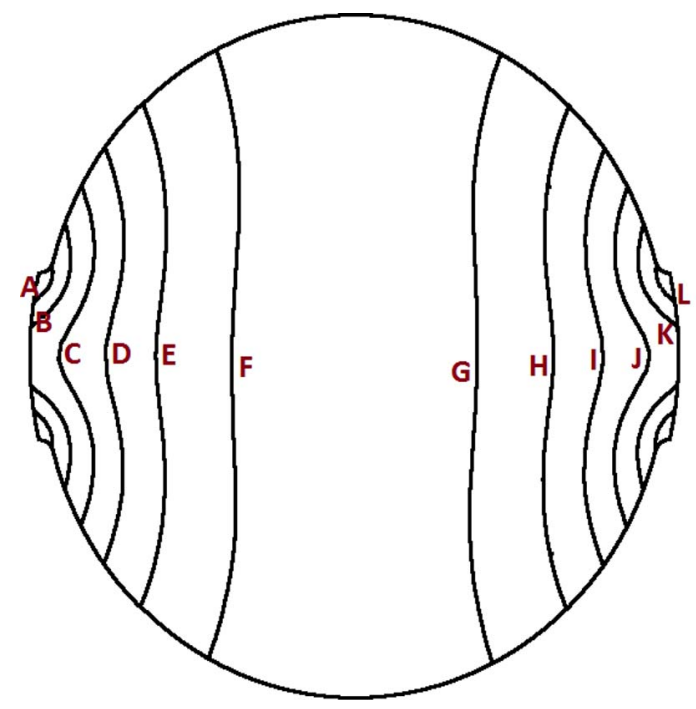

$A=-1.33 \lambda$

$B=-1.09 \lambda$

$C=-0.85 \lambda$

$D=-0.60 \lambda$

$E=-0.36 \lambda$

$F=-0.12 \lambda$

$\mathrm{G}=0.12 \lambda$

$H=0.36 \lambda$

$\mathrm{I}=0.60 \lambda$

$\mathrm{J}=0.85 \lambda$

$K=1.09 \lambda$

$L=1.33 \lambda$

Fig. 6. ANSYS analysis of the dynamical warping of the mirror at $10^{\circ}$ mechanical scan angle. The deformation amplitude is expressed in multiples of the wavelength $\lambda=510 \mathrm{~nm}$.

the longest utilized wavelength $\lambda$, with the ultimate limit being $50 \%$ [33]. In this work, the mirror is separated from the inner frame to decrease the effective stress on it, and a stiffening backside rim is used to support the mirror. The dynamic deformation along the mirror is calculated using the finite-element model, as shown in Fig. 6. About two-thirds of the mirror surface is usable for diffraction limited imaging as most of the deformation is near the edges. Note that, while the maximum peak-to-valley dynamic deformation is $2.66 \lambda$ across the mirror, once the tilt is removed, the effective dynamic deformation will be about $0.45 \lambda=230 \mathrm{~nm}$ (a factor of six reductions) according to the theory [10]. The acceptable level should be about $0.25 \lambda$ and can be achieved by further engineering of the backside stiffening rib.

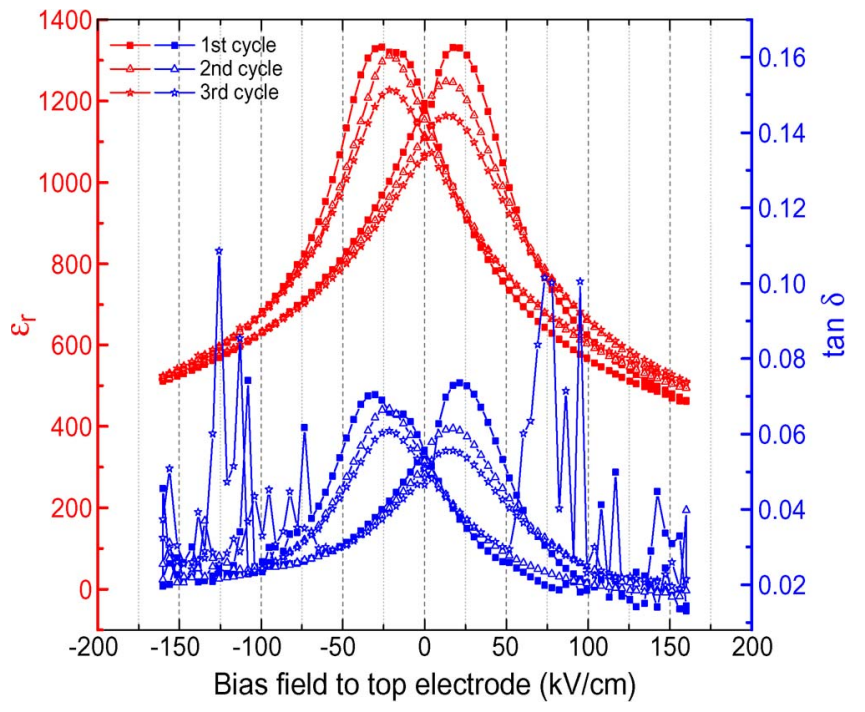

Fig. 7. $C-V$ loop of the 2- $\mu$ m-thick PZT film.

\section{Characterization}

\section{A. Drive Characteristics}

The device is divided into three isolated potentials, and electrical connections are achieved by wire bonding to the isolated areas. Prior to any measurement, piezoelectric films were poled with $10 \mathrm{~V} \mathrm{\mu m}^{-1}$ at an increased temperature of $150{ }^{\circ} \mathrm{C}$ for $15 \mathrm{~min}$. All measurements referred in this paper are performed using the following dc-biased sinusoidal signals for device actuation:

$$
\begin{aligned}
& \mathrm{V}_{\mathrm{A}}=\frac{V_{0}}{2}(1+\sin \omega t) \\
& \mathrm{V}_{\mathrm{B}}=\frac{V_{0}}{2}(1-\sin \omega t)
\end{aligned}
$$

where $V_{0}$ is the peak-to-peak (p-p) actuation voltage. These signals are applied to the electrodes placed on either side of the outer frame.

\section{B. PZT Film Characterization}

An X-ray diffraction measurement showed that the $2-\mu \mathrm{m}$ PZT film is (001)-textured to over $90 \%$. The $C-C$ loop of the PZT capacitors (Fig. 7) shows typical ferroelectric characteristics with a peaking of the dielectric constant when domains are switching. PZT dielectric constant is extracted as 1200 at zero electric field. The dielectric constant is obtained by the usual relation

$$
\varepsilon_{\mathrm{PZT}}=\frac{C \cdot t_{\mathrm{PZT}}}{A \cdot \varepsilon_{0}}
$$

where $C$ is the capacitance, $\varepsilon_{0}$ is the vacuum dielectric constant, $t_{\mathrm{PZT}}$ is the thickness $(2 \mu \mathrm{m})$, and $A$ is the area of the PZT film. The utilized sol-gel route for the PZT gives an $\mathrm{e}_{31, \mathrm{f}}$ piezoelectric coefficient from 12 to $14 \mathrm{C} / \mathrm{m}^{2}$ and a $d_{33}$ piezoelectric coefficient of more than $100 \mathrm{pm} / \mathrm{V}$ [32], [34]. 


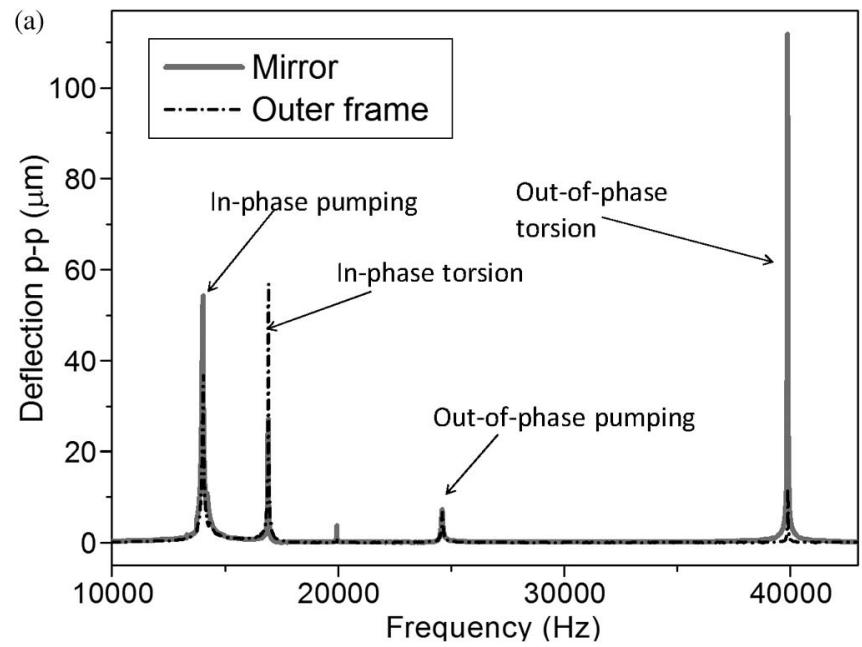

(b)

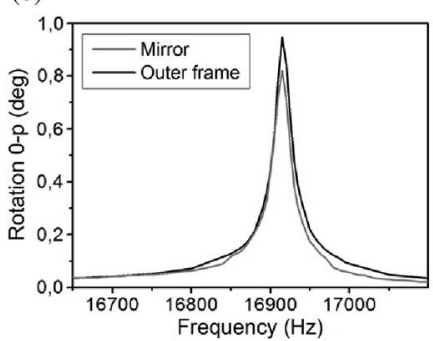

(c)

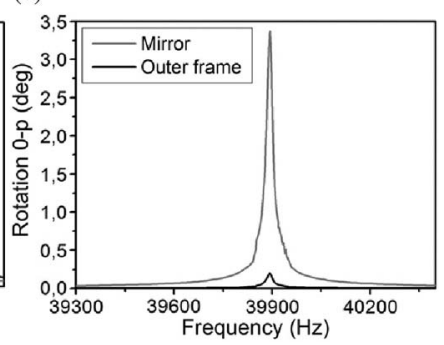

Fig. 8. (a) Frequency response curve of a fabricated device while actuated with approximately $6 \mathrm{~V}$ at $40 \mathrm{kHz}$. The graph shows the vertical (out-of-plane) deflection as a function of frequency measured with LDV at the two points shown in Fig. 2. (b) In-phase torsion mode. To better illustrate the device behavior, the deflection is presented as 0-p rotation angle. (c) Out-of-phase torsion mode, which the device is designed for. The angular coupling factor between the frames is about 17 .

\section{Scanner Results}

The PZT torsional scanner was tested to validate the mechanical-coupling mechanism under ambient pressure. The frequency response was measured with laser Doppler vibrometry (LDV) at the two measurement points shown in Fig. 2 to retrieve the motion of the outer frame and the mirror frame, respectively. For the mirror motion at the out-of-phase mode, the LDV data were corroborated with an angle measurement of a scanned laser spot. The mechanical response measured at the two points is presented in Fig. 8(a) as out-of-plane displacement. In Fig. 8(b) and (c), the in-phase and out-ofphase rotational modes (see Fig. 4) are presented in detail. For the two latter graphs, the displacement is given as mechanical zero-to-peak (0-p) rotation. The frequency response shows, as expected, a slight spring stiffening effect. There are no jump discontinuities or measurable hysteresis.

The voltage response [Fig. 9(b)] was acquired by adjusting the actuation frequency to find the maximum response at each voltage level. The peak values for each voltage were measured using an angle measurer that is aligned with the corresponding scan line, as shown in Fig. 9(a). The resonance frequency of the desired mode is around $39870 \mathrm{~Hz}$, close to the FEM prediction. At $V_{0}=24 \mathrm{~V}$, an optical scan angle of $38.5^{\circ}$ is achieved for the $D=1.4 \mathrm{~mm}$ mirror width. Corresponding to scanner comparison merits, the present scanner produces a $\theta_{\mathrm{OPT}} \times D$ of $53.9^{\circ} \times \mathrm{mm}$ at $40 \mathrm{kHz}$, leading to a $\theta_{\mathrm{OPT}} \times D \times f$ of
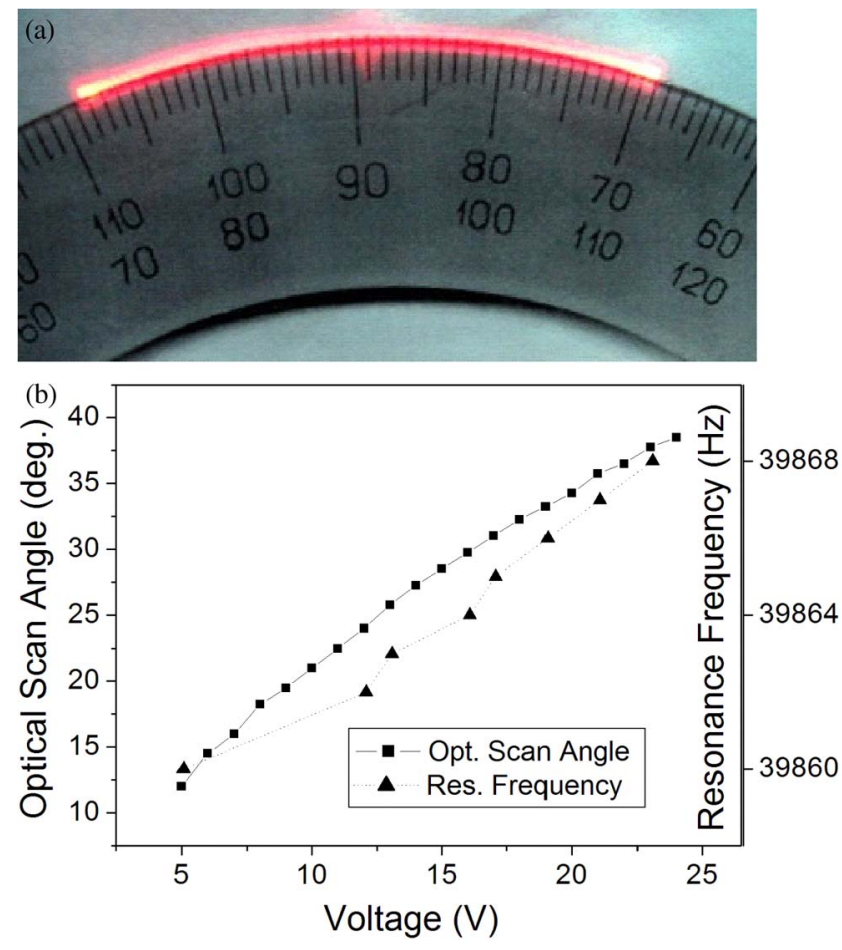

Fig. 9. (a) Scanned beam with an optical angle of $38.5^{\circ}$ at a drive voltage of $24 \mathrm{~V}$. The curvature of the scanned beam is due to the incidence angle of the laser beam onto the mirror. This effect is utilized to measure the angle via the shown angle ruler. (b) Amplitude as a function of voltage at the resonance frequency. The frequency is adjusted in steps of $1 \mathrm{~Hz}$ at each voltage level to maximize the deflection. The angle ruler above is used for the measurement. The right-hand $y$-axis shows the frequency shift for different voltages. A data point is put for each frequency increase. The frequency shift for the full sweep is only $0.02 \%$

$2156^{\circ} \times \mathrm{mm} \times \mathrm{kHz}$. Although it is a highly performing scanner, it consumes only about $28 \mathrm{~mW}$ as calculated from the equation provided in [35]

$$
P=\omega \cdot C \cdot \tan \delta \cdot V_{0}^{2}
$$

where $\omega$ is the actuation frequency, $C$ is the capacitance, $\tan \delta$ is the loss factor, and $V_{0}$ is the root mean square of the actuation voltage.

At the out-of-phase torsion mode, the outer-frame deflection is amplified and transferred to the inner frame with a mechanical-coupling gain of about 17 and agrees well with the ANSYS simulation results.

\section{Mirror Deformation Measurement}

The static and dynamic deformations of the mirror were measured using a stroboscopic white light interferometer (Bruker, ContourGT In-Motion). As previously explained, 2- $\mu$ m oxide is left below the mirror metal to shorten the fabrication process. The p-p static deformation of about $0.13 \mu \mathrm{m}$ induced by the oxide is shown in Fig. 10. The static deformation can be eliminated by removing the oxide layer. The dynamic deformation of the mirror due to acceleration forces is calculated by subtracting the static deformation from the measured dynamic deformation at $4^{\circ}$ mechanical angle ( $16^{\circ}$ optical scan angle). As shown in Fig. 11, the p-p dynamic deformation is about $130 \mathrm{~nm}$. At larger scan angles, fringes are blurred, and data are 


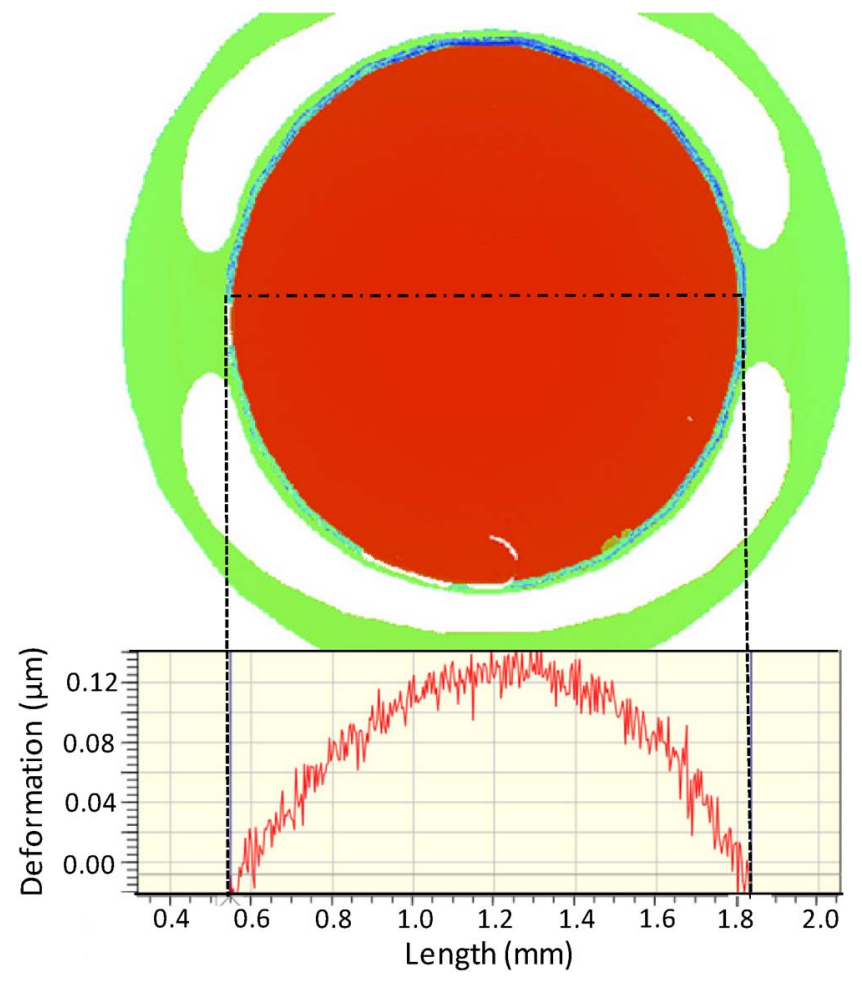

Fig. 10. White light interferometer measurement of the mirror at rest.

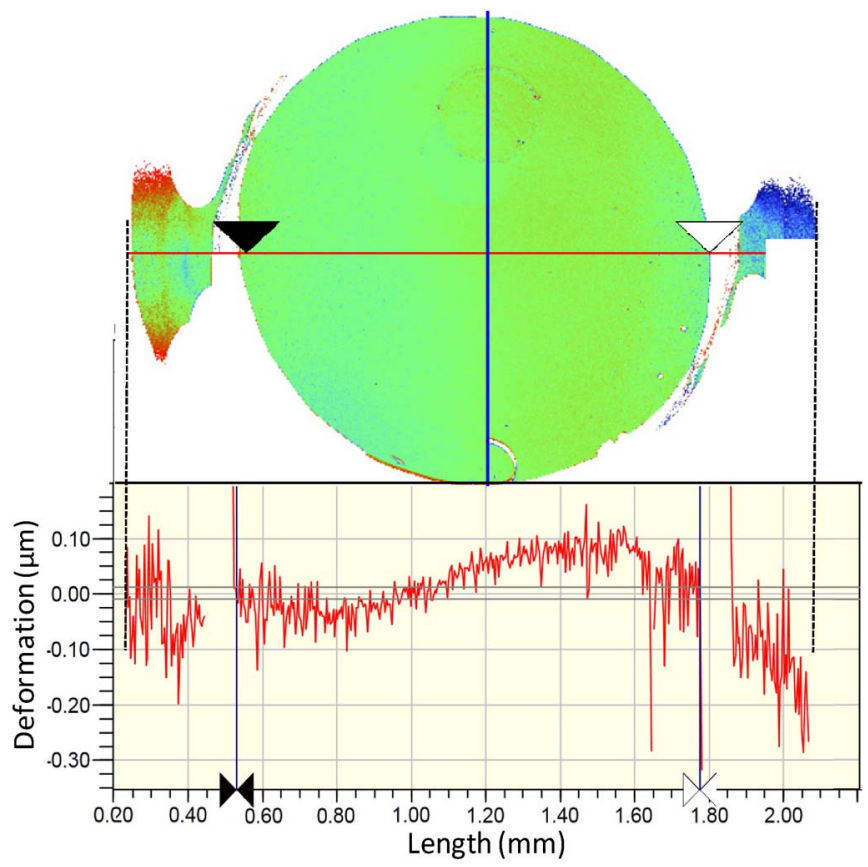

Fig. 11. Dynamic deformation calculated by subtracting the mirror curvature at rest from the measured shape at $16^{\circ}$ optical scan angle. The optically relevant p-p deformation is about $130 \mathrm{~nm}$.

noisier. Since the deformation is linear with the scan angle, the dynamic deformation can be estimated as $325 \mathrm{~nm}$ at $10^{\circ}$ mechanical angle, which agrees reasonably well with the 230-nm estimation using the FEM model.

\section{E. Angle Sensor Results}

Since the phase is very sensitive close to resonance, a repeatable scan line requires angle feedback to meet the demands of

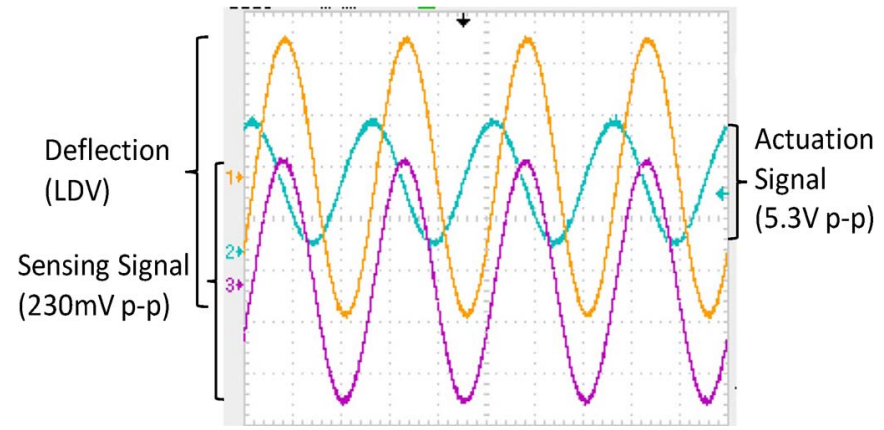

Fig. 12. Example of the sensing signal while the device is vibrating at resonance with a mechanical zero-to-peak torsion angle of $1.6^{\circ}$. The device used for this experiment has segmented electrodes; only one fourth of the total PZT area is used for actuation, and one fourth is used for sensing.

dynamic displays. A version of the PZT where each side of the PZT is segmented into two separate electrodes was fabricated to test the angle sensor concept. One half of the electrode in each side is used to drive the scanner, and the other half is used for the scan angle sensing. Proof of concept experimental results is shown in Fig. 12.

The readout signal is very clear and at $230-\mathrm{mV}$ level. The sensing signal is $4.3 \%$ of the input voltage, which translates into very good conversion efficiency from the electrical to mechanical and back to electrical energy domain. The phase of the sensing signal follows the deflection signal measured with an LDV fully, with a constant small phase shift. PZT is generally known to have some hysteresis; however, since the stress on the film is bidirectional and symmetrical, the sensor signal is also bidirectional and does not show any hysteresis. Future work will focus on engineering the size and location of the PZT angle sensor across the scanner to minimize the impact on the actuator area.

\section{CONCLUSion AND Discussion}

A high-performance PZT resonant scanner has been designed, fabricated, and tested. A novel MEMS scanner solution, combining the mechanical coupling principle and PZT thin-film actuation, has been proposed to achieve large optical angles at high frequencies. Analytical reasoning and a FEM have been developed and validated with experimental results. The present scanner is the best-performing one in the literature in terms of the product of mirror size $(1.4 \mathrm{~mm})$, optical scan angle $\left(38.5^{\circ}\right)$, and resonance frequency of $39870 \mathrm{~Hz}$. In addition to this record performance, it consumes only about $28-\mathrm{mW}$ power, which is little compared to other actuation schemes. Dynamic deformation of the mirror is taken under control by separating it from the inner frame and using backside reinforcement rim underneath the mirror. Moreover, preliminary PZT sensing experiments are done by separating PZT layers for actuation and sensing purposes.

The presented scanner's performance meets the frequency requirements and with further work will be able to meet all the requirements of full HD resolution picoprojectors working at ambient conditions. Vacuum packaging always comes with a considerable cost and is very desirable to avoid. This record performance can be attributed to ingenuity in the mechanical design, as well as expertise in the PZT and the MEMS process. 


\section{ACKNOWLEDGMENT}

The authors would like to thank Y. Leblebici and the CMI staff at EPFL, Switzerland, where microfabrication was performed; A. Mazzalai and N. Chidambaram for their help in PZT thin-film processing and integration; M. Helsel for his help with device fabrication; and S. Ölçer, E. Ermek, and N. Pelin Ayerden for their help with packaging and measurements.

\section{REFERENCES}

[1] U. Baran, D. Brown, S. Holmstrom, D. Balma, W. O. Davis, A. Mazzalai, P. Muralt, and H. Urey, "High frequency torsional MEMS scanner for displays," in Proc. 25th Annu. IEEE MEMS, Paris, France, 2012, pp. 636-639.

[2] A. D. Yalcinkaya, H. Urey, D. Brown, T. Montague, and R. Sprague, "Two-axis electromagnetic microscanner for high resolution displays," J. Microelectromech. Syst., vol. 15, no. 4, pp. 786-794, Aug. 2006.

[3] J. Tauscher, W. O. Davis, D. Brown, M. Ellis, Y. Ma, M. E. Sherwood, D. Bowman, M. P. Helsel, S. Lee, and J. W. Coy, "Evolution of MEMS scanning mirrors for laser projection in compact consumer electronics," in Proc. SPIE, Jan. 2010, vol. 7594, pp. 75940A-1-75940A-12.

[4] A. Q. Liu and X. M. Zhang, "A review of MEMS external-cavity tunable lasers," J. Micromech. Microeng., vol. 17, no. 1, pp. R1-R13, Jan. 2007.

[5] M. C. Wu, O. Solgaard, and J. E. Ford, "Optical MEMS for lightwave communication," J. Lightw. Technol., vol. 24, no. 12, pp. 4433-4454, Dec. 2006.

[6] C. Lee and J. A. Yeh, "Development and evolution of MOEMS technology in variable optical attenuators," J. Micro/Nanolith. MEMS MOEMS, vol. 7, no. 2, pp. 021003-1-021003-16, Apr.-Jun. 2008.

[7] C. Ataman, H. Urey, and A. Wollter, "A Fourier transform spectrometer using resonant vertical comb actuators," J. Micromech. Microeng., vol. 16, no. 12, pp. 2517-2523, Dec. 2006.

[8] J. Sun, S. Guo, L. Wu, L. Liu, S.-W. Choe, B. S. Sorg, and H. Xie, "3D in vivo optical coherence tomography based on a low-voltage, large-scanrange 2D MEMS mirror," Opt. Exp., vol. 18, no. 12, pp. 12 065-12 075, Jun. 2010.

[9] W. O. Davis, D. Brown, M. Helsel, R. Sprague, G. Gibson, A. Yalcinkaya, and $\mathrm{H}$. Urey, "High-performance silicon scanning mirror for laser printing," in Proc. SPIE, Jan. 2007, vol. 6466, pp. 64660D-1-64660D-7.

[10] H. Urey, D. W. Wine, and T. D. Osborn, "Optical performance requirements for MEMS-scanner based microdisplays," in Proc. SPIE, Sep. 2000, vol. 4178, pp. 176-185.

[11] K. Yamada and T. Kuriyama, "A novel asymmetric silicon micro-mirror for optical scanning display," in Proc. 11th Anпи. Int. Workshop Microelectromech. Syst., Heidelberg, Germany, 1998, pp. 110-115.

[12] T. Iseki, M. Okumura, and T. Sugawara, "Shrinking design of a MEMS optical scanner having four torsion beams and arms," Sens. Actuators A, Phys., vol. 164, no. 1/2, pp. 95-106, Nov./Dec. 2010.

[13] P. Muralt, "Ferroelectric thin films for micro-sensors and actuators: A review," J. Micromech. Microeng., vol. 10, no. 2, pp. 136-146, Jun. 2000.

[14] P. Muralt, M. Kohli, T. Maeder, A. Kholkin, K. Brooks, N. Setter, and R. Luthier, "Fabrication and characterization of PZT thin-film vibrators for micromotors," Sens. Actuators A, Phys., vol. 48, no. 2, pp. 157-165, May 1995.

[15] P. Muralt, N. Ledermann, J. Paborowski, A. Barzegar, S. Gentil, B. Belgacem, S. Petitgrand, A. Bosseboeuf, and N. Setter, "Piezoelectric micromachined ultrasonic transducers based on PZT thin films," IEEE Trans. Ultrason., Ferroelectr., Freq. Control, vol. 52, no. 12, pp. 22762288, Dec. 2005.

[16] T. Fujii, S. Watanabe, M. Suzuki, and T. Fujiu, "Application of lead zirconate titanate thin film displacement sensors for the atomic force microscope," J. Vac. Sci. Technol. B, Microelectron. Nanometer Struct., vol. 13, no. 3, pp. 1119-1122, May 1995.

[17] M. Lebedev, H. Sato, and J. Akedo, "Optical micro-scanner fabricated on stainless steel by aerosol deposition method," in Proc. IEEE Int. Conf. Ultrason., Ferroelect., Freq. Control, Aug. 2004, pp. 165-168.

[18] J. G. Smits, K. Fujimoto, and V. F. Kleptsyn, "Microelectromechanical flexure PZT actuated optical scanner: Static and resonance behavior," J. Micromech. Microeng., vol. 15, no. 6, pp. 1285-1293, Jun. 2005.

[19] F. Filhol, E. Defaÿ, C. Divoux, C. Zinck, and M. T. Delaye, "Resonant micro-mirror excited by a thin-film piezoelectric actuator for fast optical beam scanning," Sens. Actuators A, Phys., vol. 123/124, pp. 483-489, Sep. 2005 .
[20] J. H. Park, J. Akedo, and H. Sato, "High-speed metal-based optical microscanners using stainless-steel substrate and piezoelectric thick films prepared by aerosol deposition method," Sens. Actuators A, Phys., vol. 135, no. 1, pp. 86-91, Mar. 2007.

[21] T. Iseki, M. Okumura, and T. Sugawara, "High speed and wide angle deflection optical MEMS scanner using piezoelectric actuation," IEEJ Trans. Elect. Electron. Eng., vol. 5, no. 3, pp. 361-368, May 2010.

[22] S. Matsushita, I. Kanno, R. Yokokawa, and H. Kotera, "Metal-based piezoelectric MEMS scanner mirrors composed of PZT thin films on titanium substrates," in Proc. 16th Int. Solid-State Sens., Actuators Microsyst. Conf., Beijing, China, 2011, pp. 574-577.

[23] Y. Yasuda, M. Akamatsu, M. Tani, T. Iijima, and H. Toshiyoshi, "Piezoelectric 2D-optical micro scanners with PZT thick films," Integr. Ferroelect., vol. 76, no. 1, pp. 81-91, Jan. 2005.

[24] T. Kobayashi, R. Maeda, and T. Itoh, "Low speed piezoelectric optical microscanner actuated by piezoelectric microcantilevers using $\mathrm{LaNiO}_{3}$ buffered $\mathrm{Pb}(\mathrm{Zr}, \mathrm{Ti}) \mathrm{O}_{3}$ thin film," Smart Mater. Struct., vol. 18, no. 6, p. 065008, Jun. 2009.

[25] K. H. Koh, T. Kobayashi, J. Xie, A. Yu, and C. Lee, "Novel piezoelectric actuation mechanism for a gimbal-less mirror in 2D raster scanning applications," J. Micromech. Microeng., vol. 21, no. 7, p. 075001, Jul. 2011.

[26] M. Tani, M. Akamatsu, Y. Yasuda, H. Fujita, and H. Toshiyoshi, "A combination of fast resonant mode and slow static deflection of SOI-PZT actuators for MEMS image projection display," in Proc. IEEE/LEOS Opt. MEMS, Big Sky, MT, 2006, pp. 25-26.

[27] T. Iseki, M. Okumura, T. Sugawara, and M. K. Kurosawa, "Deflection properties of a MEMS optical scanner with four torsion beams and L-shaped arms," Sens. Actuators A, Phys., vol. 178, pp. 154-163, May 2012.

[28] U. Baran, M. Tani, M. Akamatsu, Y. Yasuda, H. Fujita, and H. Toshiyoshi, "A built-in vibration sensor using arc-discharged reactive ion plated PZT," IEEJ Trans. Sens. Micromach., vol. 131, no. 3, pp. 128-129, Mar. 2011.

[29] C. Lee, T. Itoh, T. Ohashi, R. Maeda, and T. Suga, "Development of a piezoelectric self-excitation and self-detection mechanism in PZT microcantilevers for dynamic scanning force microscopy in liquid," J. Vac. Sci. Technol. B, Microelectron. Nanometer Struct., vol. 15, no. 4, pp. 15591563, Jul. 1997.

[30] A. Arslan, D. Brown, W. Davis, S. Holmstrom, K. S. Gokce, and H. Urey, "Comb-actuated resonant torsional microscanner with mechanical amplification," J. Microelectromech. Syst., vol. 19, no. 4, pp. 936-943, Aug. 2010.

[31] P. Muralt, T. Maeder, L. Sagalowicz, S. Hiboux, S. Scalese, D. Naumovic, R. G. Agostino, N. Xanthopoulos, H. J. Mathieu, L. Patthey, and E. L. Bullock, "Texture control of $\mathrm{PbTiO}_{3}$ and $\mathrm{Pb}(Z r, T i) O_{3}$ thin films with $\mathrm{TiO}_{2}$ seeding," J. Appl. Phys., vol. 83, no. 7, pp. 3835-3841, Apr. 1998.

[32] F. Calame and P. Muralt, "Growth and properties of gradient free solgel lead zirconate titanate thin films," Appl. Phys. Lett., vol. 90, no. 6 , pp. 062907-1-062907-3, Feb. 2007.

[33] H. Urey and D. Dickensheets, "MOEMS and applications," in Display and Imaging Systems, E. Motamedi, Ed. Bellingham, WA: SPIE, 2005, ch. 8 .

[34] N. Ledermann, P. Muralt, J. Baborowski, S. Gentil, K. Mukati, M. Cantoni, A. Seifert, and N. Setter, " $\left\{\begin{array}{lll}1 & 0 & 0\end{array}\right\}$-Textured, piezoelectric $\mathrm{Pb}$ (Zrx, Ti1-x) O3 thin films for MEMS: Integration, deposition and properties," Sens. Actuators A, Phys., vol. 105, no. 2, pp. 162-170, Jul. 2003.

[35] N. G. Ledermann, Piezoelectric Acoustic Sensors and Ultrasonic Transducers Based on Textured PZT Thin Films. Lausanne, Switzerland: École Polytechnique Fédérale de Lausanne, 2003.

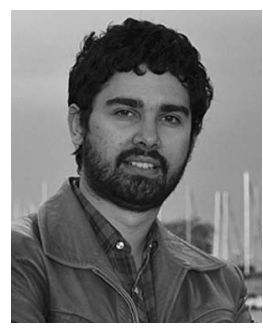

Utku Baran (S'07) received the B.Sc. degree in electrical and electronics engineering (with meritbased scholarship) in 2010 from Koç University, Istanbul, Turkey, where he is currently working toward the M.Sc. degree in the Department of Electri$\mathrm{cal}$ and Electronics Engineering.

$\mathrm{He}$ was an Undergraduate Researcher with the Optical Microsystems Laboratory, Koç University, for three years. He has been with Microvision Inc., Redmond, WA; The University of Tokyo, Tokyo, Japan; North Carolina State University, Raleigh; and Aselsan as an Intern. He also spent four months at EPFL-Lausanne, Switzerland, as a Visiting Researcher. He is the author of more than five journal and conference proceedings papers. He is the holder of two pending patents. His research interests include optical microelectromechanical systems and scannerbased display systems. 


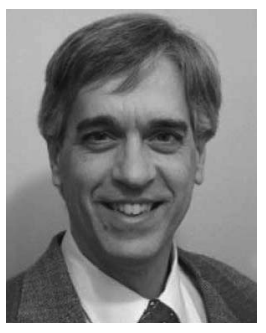

Dean Brown received the B.S. degree in mechanical engineering from the University of California, Santa Barbara, in 1974.

He was with Bunker-Ramo, Trumbull, CT, and Honeywell, Morristown, NJ, working in ocean systems technology from 1974 to 1981 and developing deep-water packaging and deployment systems for sonobuoys and mechanical systems for a submersible remotely operated underwater vehicle. From 1981 to 1997 , he was with Chem-Nuclear Systems, JLR Computer Analysis and Packaging Technology, where he performed advanced design analyses of mechanical systems in a variety of industries, including chemical and radioactive waste handling, electronics packaging, magnetic systems, heavy trucking, nuclear fuel rod and power plant systems, lifting and handling equipment, marine systems, tunnel-boring equipment, paper-mill equipment, and sports and fitness equipment. Since 1997, he has been with Allied Signal and Microvision, Inc., Redmond, WA, where he has designed and analyzed silicon MEMS devices, including gyroscopic sensors and scanning mirrors. $\mathrm{He}$ is the holder of nine patents related to MEMS scanning mirrors.

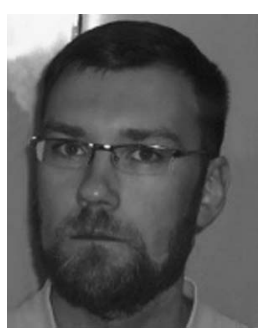

Sven Holmstrom received the M.Sc. degree in engineering biology from Linköping University, Linköping, Sweden in 2005.

Since 2005, he has been a Research Engineer with the Optical Microsystems Laboratory, Department of Electrical and Electronics Engineering, Koç University, Istanbul, Turkey. During his time there, he has spent about one year as a Visiting Researcher at the École Polytechnique Fédérale de Lausanne, Lausanne, Switzerland, and four months at Chalmers University of Technology, Gothenburg, Sweden. He is the coauthor of nine journal and 15 conference proceedings papers. He is the holder of a pending patent. His research interests include microfabrication and micro-optoelectromechanical systems.

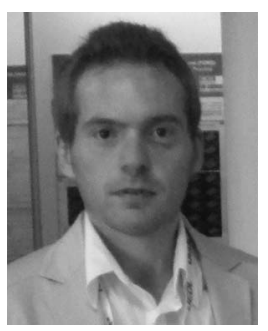

Davide Balma received the B.Sc. degree in physics and the M.Sc. degree in physics of advanced technologies from the University of Turin, Turin, Italy, in 2006 and 2008, respectively, and the Ph.D. degree in electronic devices from the Polytechnic of Turin, Turin, in 2012.

He conducted most of his research activity at Xlab, Materials and Microsystems Laboratory, Department of Materials Science and Chemical Engineering, Polytechnic of Turin. During this period, he spent six months at the Ceramics Laboratory, École Polytechnique Fédérale de Lausanne (EPFL), Lausanne, Switzerland. Since 2012, he has been a Postdoctoral Researcher with the Ceramic Laboratory, Department of Material Science, EPFL. His research interests include MEMS fabrication technologies and growth and characterization of lead zirconate titanate thin films.

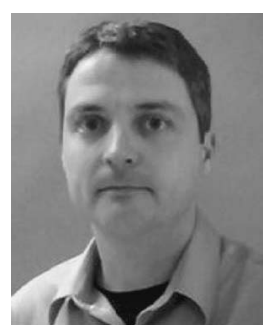

Wyatt O. Davis (M'08) received the B.S. and M.S. degrees from Washington State University, Pullman, in 1993 and 1995, respectively, and the Ph.D. degree from the University of California, Berkeley, in 2001, all in mechanical engineering.

From 2001 to 2003, he was a MEMS Engineer with Onix Microsystems, Richmond, CA. Since 2003, he has been with Microvision, Inc., Redmond, WA, where he is currently a Principal Engineer, developing MEMS scanners for display and imaging applications. He is the holder of 11 issued patents on MEMS scanning mirror technology.

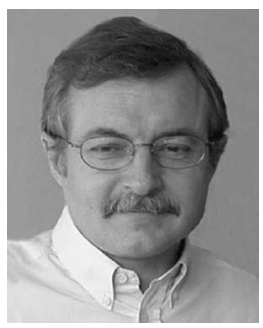

Paul Muralt (M'95-SM'05) received the Diploma in experimental physics and the Ph.D. degree from the Swiss Federal Institute of Technology (ETH), Zurich, Switzerland, in 1978 and 1984, respectively. $\mathrm{He}$ developed his Ph.D. thesis in the Solid State Laboratory of ETH.

$\mathrm{He}$ is currently a Professor in the Department of Material Science, Ecole Polytechnique Federale de Lausanne (EPFL), Lausanne, Switzerland. In 1984 and 1985 , he held a postdoctoral position at the IBM Research Laboratory, Zurich, where he pioneered the application of scanning tunneling microscopy to surface potential imaging. After a stay at the Free University of Berlin, Berlin, Germany, he joined the Balzers group in Liechtenstein in 1987. He specialized in sputter deposition techniques and, beginning in 1991, managed a department for development and applications of physical vapor deposition processes. In 1993, he joined the Ceramics Laboratory, EPFL, where he started MEMS activities as a group leader for thin-film and device research. His research interests are thin-film growth and integration issues of ferroelectric and other polar materials; property-microstructure relationships; and applications of polar materials in semiconductor, microelectromechanical, and nanoscale devices. As a teacher, he lectures on thin-film deposition, micropatterning, and ceramics. He has authored or coauthored more than 180 scientific articles.

Mr. Muralt is a member of the Materials Research Society.

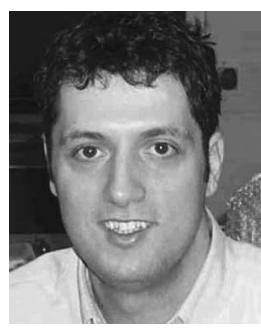

Hakan Urey (M'92-SM'09) received the B.S. degree from Middle East Technical University, Ankara, Turkey, in 1992, and the M.S. and Ph.D. degrees from Georgia Institute of Technology, Atlanta, in 1996 and 1997, respectively, all in electrical engineering.

After completing his Ph.D. studies, he joined Microvision Inc., Seattle, WA, as a Research Engineer, and played a key role in the development of scanning display technologies. He was the Principal System Engineer when he left Microvision to join the Faculty of Engineering, Koç University, Istanbul, Turkey, where he established the Optical Microsystems Research Laboratory. He is currently a Professor of electrical engineering at Koç University. He is the holder of 23 issued patents and more than ten pending patents. He is the author or coauthor of 40 journal and 80 conference proceedings papers, six edited books, and four book chapters. His research interests are optical MEMS, optical system design, and laser-based 2-D and 3-D display and imaging systems. 